\title{
Children with Epilepsy in the Era of COVID-19 Pandemic
}

\author{
Mahmoud Reza Ashrafi ${ }^{1}$ Mahmoud Mohammadi ${ }^{1}$ Reza Shervin Badv ${ }^{1}$ Golamreza Zamani ${ }^{1}$ \\ Ali Reza Tavasoli ${ }^{1}$ Morteza Heidari ${ }^{1}$ Reza Azizimalamiri ${ }^{2}$
}

\author{
${ }^{1}$ Division of Paediatric Neurology, Department of Paediatrics, Growth \\ and Development Research Center, Children's Medical Centre, \\ Paediatrics Centre of Excellence, Tehran University of Medical \\ Sciences, Tehran, Iran \\ 2 Department of Paediatric Neurology, Golestan Medical, Educational, \\ and Research Center, Ahvaz Jundishapur University of Medical \\ Sciences, Ahvaz, Iran \\ J Pediatr Epilepsy 2020;9:53-54.
}

After the first report of several cases of pneumonia of unknown causes that resulted in acute respiratory distress syndrome in Wuhan, China in December 2019, a novel coronavirus was identified as the causative agent. ${ }^{1}$ This was the seventh strain of human coronaviruses and was later named severe acute respiratory syndrome coronavirus 2 by the World Health Organization, and the disease caused by it was called coronavirus disease 2019 (COVID-19). ${ }^{2}$ On March 11, 2020, the World Health Organization declared COVID-19 to be a pandemic. ${ }^{2}$ Although the COVID-19 virus can infect human beings from the newborn period to old age, fortunately this virus affects children less frequently than adults, has no severe signs and symptoms, and also has lower morbidity and mortality. Unfortunately, it seems that COVID-19 is going to live with us at least until an effective vaccine becomes available, which is estimated to be over a year in an optimistic view. Therefore, pediatric neurologists have to modify their previous routine practices to provide appropriate care to patients against this potentially life-threatening infection.

Epilepsy is one of the most frequent neurologic disorders in children. Although the majority of patients with childhood-onset epilepsy attain remission, about one-third of these children develop antiseizure drug (ASD) resistance and need more sophisticated measures including surgery. ${ }^{3}$

Immunologic abnormalities have been reported in epilepsy either by direct effect of epilepsy on the immune system or by effect of ASDs on serum immunoglobulin levels. ${ }^{4-6}$ There are several reports concerning the effects of ASDs therapy on humoral and cellular immunity. Several documented studies have shown that antiepileptic drugs can decrease serum immunoglobulin levels in children treated with ASDs. ${ }^{7-10}$ The most common reported reduced immunoglobulin is

received

April 20, 2020

accepted after revision

April 28, 2020

published online

May 22, 2020

Address for correspondence Reza Azizimalamiri, MD, Department of Paediatric Neurology, Golestan Medical, Educational, and Research Center, Ahvaz Jundishapur University of Medical Sciences, 90, Golestan Boulevard, Ahvaz 6135733118, Iran

(e-mail: azizi.ramin@gmail.com; azizi-r@ajums.ac.ir).

immunoglobulin (Ig)A, especially with phenytoin treatment. Carbamazepine and sodium valproate are also associated with changes in both humoral and cellular immunity. Furthermore, some studies reported that the reduction of serum immunoglobulins caused by ASDs is associated with increased susceptibility to recurrent infections. Ashrafi et al measured serum IgA, IgG, and IgM levels in a cohort of epileptic patients compared with age and gender-matched controls. This study indicated that ASDs could reduce serum immunoglobulins, especially IgA and IgG; among them, the carbamazepine effect is of more concern. ${ }^{6}$ There were few studies about ASDs effects on serum IgG subclasses. IgG subclass deficiency induced by ASDs predisposes patients to specific infections, whereas asymptomatic patients were also reported.

One of the main issues in children with epilepsy, especially those with drug resistance, is anticonvulsant therapy and the possible interactions between antiseizure medications and the agents that might be used for the treatment of COVID-19 in these patients. Fortunately, a practical manual addressing this concern has been produced and frequently updated by the Liverpool Drug Interaction Group (based at the University of Liverpool, United Kingdom), in collaboration with the University Hospital of Basel (Switzerland) and Radboud University Medical Center (Netherlands). ${ }^{11}$ On page 7 of this manual, the interactions between anticonvulsants and the agents that might be used in the treatment of COVID19 have been explained. This manual (last updated: April 9, 2020) could be very useful when we need to administer ASDs or to change their dosages in COVID-19 patients. Moreover, no evidence-based recommendation could be found around this issue of how often patients should go to their neurologist for control during this pandemic.

Copyright $\odot 2020$ by Georg Thieme Verlag KG, Stuttgart · New York
DOI https://doi.org/ 10.1055/s-0040-1712497. ISSN 2146-457X. 
Until now, no study has shown that children with epilepsy without any health problems and easily controlled seizures seem to have a higher risk of getting coronavirus. Children with epilepsy who suffer from health problems like swallowing dysfunction, drooling, chest deformities, and those taking benzodiazepine are at a larger risk for respiratory infections that might put them at higher risk for COVID-19. In addition, in some epileptic syndromes taking medicines for controlling seizures may affect the immune system such as adrenocorticotropic hormone, steroids, and immunotherapies. Moreover, $30 \%$ of children with epilepsy are drug-resistant and need rational polytherapy with ASDs that have already been shown to lead to humoral immunosuppression. ${ }^{4-6}$ Therefore, it is highly likely that a proportion of children with drug-resistant epilepsy, such as those with concurrent health problems, may be at higher risk of developing more severe symptoms with viral illnesses like COVID-19.

In contrast to our opinion around the children with epilepsy, especially those with concurrent health problems and those with drug resistance, Professor Ley Sander-the Medical Director at the Epilepsy Society in the United Kingdom-has different opinions for patients with epilepsy. ${ }^{12} \mathrm{We}$, authors of this article, appreciate his comments and we think that many people with epilepsy need to consider his recommendations. However, we believed that there are many unanswered questions about COVID-19 in children with epilepsy and it is logical that we would have concerns about these patients.

In conclusion, potentially a proportion of the children with epilepsy, especially those with concurrent health problems and drug-resistant epilepsy, may be at higher risk in the era of COVID-19. Therefore, it is indicated that all the child neurologists and caregivers do apply official guidelines to reduce the chance of COVID-19 in these vulnerable children.
Conflict of Interest

None declared.

\section{References}

1 Lu H, Stratton CW, Tang YW. Outbreak of pneumonia of unknown etiology in Wuhan, China: the mystery and the miracle. J Med Virol 2020;92(04):401-402

2 Cucinotta D, Vanelli M. WHO declares COVID-19 a pandemic. Acta Biomed 2020;91(01):157-160

3 Camfield CS, Camfield PR, Gordon K, Wirrell E, Dooley JM. Incidence of epilepsy in childhood and adolescence: a population-based study in Nova Scotia from 1977 to 1985. Epilepsia 1996;37(01):19-23

4 Aarli JA. Changes in serum immunoglobulin levels during phenytoin treatment of epilepsy. Acta Neurol Scand 1976;54(05): 423-430

5 Ashrafi M, Hosseini SA, Abolmaali S, et al. Effect of anti-epileptic drugs on serum immunoglobulin levels in children. Acta Neurol Belg 2010;110(01):65-70

6 Ashrafi MR, Hosseini SA, Biglari M, et al. Effect of anti-epileptic drugs on serum level of IgG subclasses. Iran J Pediatr 2010;20(03):269-176

7 Bardana EJ Jr, Gabourel JD, Davies GH, Craig S. Effects of phenytoin on man's immunity. Evaluation of changes in serum immunoglobulins, complement, and antinuclear antibody. Am J Med 1983;74 (02):289-296

8 Gilhus NE, Strandjord RE, Aarli JA. The effect of carbamazepine on serum immunoglobulin concentrations. Acta Neurol Scand 1982; 66(02):172-179

9 Go T. Carbamazepine-induced IgG1 and IgG2 deficiency associated with B cell maturation defect. Seizure 2004;13(03):187-190

10 Ranua J, Luoma K, Auvinen A, et al. Serum IgA, IgG, and IgM concentrations in patients with epilepsy and matched controls: a cohort-based cross-sectional study. Epilepsy Behav 2005;6(02): 191-195

11 COVID-19 drug interactions. Available at: https://www.covid19druginteractions.org/2020. Accessed April 26, 2020

12 And E. The coronavirus (COVID-19) FAQs. Available at: https:// www.epilepsysociety.org.uk/epilepsy-and-coronavirus-covid-19 -faqs\#.XqXY5mgzaHs2020. Accessed April 26, 2020 